\title{
Biomimetic Brønsted Acid-Catalyzed Carbonyl-Olefin Metathesis Enabled by Hydrogen Bonding Networks
}

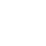

\author{
Tuong Anh To, ${ }^{\dagger}$ Chao Pei, ${ }^{\S}$ Rene M. Koenigs, ${ }^{\circledR}$ Thanh Vinh Nguyen** $^{\dagger}$ \\ ${ }^{+}$School of Chemistry, University of New South Wales, Sydney, NSW 2052, Australia \\ SInstitute of Organic Chemistry, RWTH Aachen, Aachen D52074, Germany
}

\section{Biomimetic catalyst activation by hydrogen bonds}

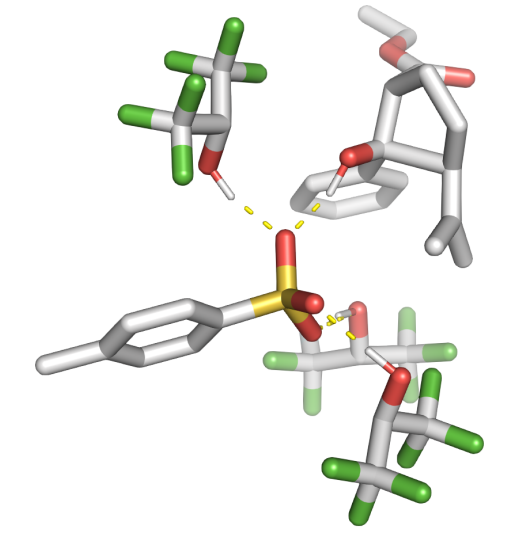
Hydrogen bond complex of pTSA and HFIP and substrate in carbonyl olefin metathesis reactions

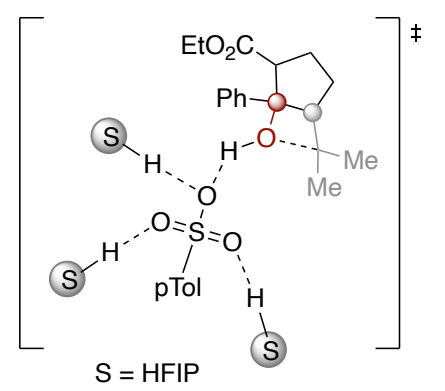

- Hydrogen bond network between catalyst and multiple molecules of HFIP

- Increased catalytic efficiency of Brønsted acid catalyst and stabilize reaction intermediates

- Mechanism of action revealed by experimental and DFT studies

Abstract: Synthetic chemists have learned to mimic nature in using hydrogen bonds and other weak interactions to dictate the spatial arrangement of reaction substrates and to stabilize transition states to enable highly efficient and selective reactions. The activation of a catalyst molecule itself by hydrogen bonding networks, in order to control its catalytic activity to achieve desired reaction outcomes is much less explored in organic synthesis, despite being a common strategy in nature. Herein, we show our investigation into this underexplored area by studying the promotion of carbonylolefin metathesis reactions by hydrogen bonding-assisted Brønsted acid catalysis. The carbonyl-olefin metathesis reaction has recently emerged as a powerful synthetic tool for functional group interconversion of carbonyls and alkenes. However, the application of Brønsted acid catalysts in carbonyl-olefin metathesis reaction, especially in homogeneous conditions, remains scarce and poorly understood. In this work, we report the use of hexafluoroisopropanol solvent in combination with para-toluenesulfonic acid to efficiently catalyze carbonyl-olefin metathesis reactions. Our experimental and computational mechanistic studies reveal not only an interesting role of HFIP solvent in assisting this Brønsted acid catalyzed reaction but also insightful knowledge about the current limitations of the carbonyl-olefin metathesis reaction. 
Weak non-covalent interactions take up an essential role in chemistry and biology and form the basis for the assembly of complex supramolecular structures in natural and artificial systems. ${ }^{1}$ Among them, the hydrogen bond is of unique importance and indispensable for the formation of entities essential for living, such as proteins or nucleic acids. ${ }^{2}$ In artificial systems, the hydrogen bond is key for the assembly of supramolecular structures, catalyst design, materials, molecular recognition and machinery. ${ }^{1}$ To realize such human-designed systems, chemists often mimic nature in using hydrogen bonds to dictate the spatial arrangement of individual molecules in supramolecular assemblies ${ }^{3}$ or to stabilize transition states in catalysis to enable highly efficient and selective reactions. ${ }^{4-6}$ The famous Hajos-Parrish-Eder-Sauer-Wiechert reaction is such an example that founded the underlying concept for modern organocatalysis. ${ }^{7,8}$

One of the longest standing paradigms in catalysis lies within the activation of reaction substrates with hydrogen-bonding catalysts, which also are small organic molecules themselves. ${ }^{9,10}$ Numerous hydrogen-bonding motifs have been reported to date and the Corey, Schreiner or Takemoto catalysts (shown in Scheme 1B1) represent a few versatile and well-explored examples of such systems. Nonetheless, the activation of a catalyst molecule itself by hydrogen bonding is much less explored in organic synthesis, despite being a common occurrence in nature. ${ }^{2,6}$ For instance, hydrogen bonds between amino acids side chains play a key role to enhance catalytic activity of enzymes to facilitate reactions at ambient conditions. ${ }^{6}$ A prominent example can be found in serine proteases as demonstrated in Scheme 1A. ${ }^{11}$ In this case, a hydrogen bond network between three amino acid sites, or the so-called catalytic triad, has been identified as vital for its catalytic function and to enhance the nucleophilicity of serine to allow for scission of amide bonds. ${ }^{12}$

In recognition of such an underexplored area in chemistry, we hypothesized that catalyst activation by hydrogen bond networks could be achieved using small molecules in a biomimetic fashion. This strategy would be useful as very reactive catalysts are efficient for the desired chemical transformation but often promote unwanted side-reactions at the same time. ${ }^{13}$ By employing a moderately or poorly active catalyst to ensure better selectivity, and enhancing its efficacy by hydrogen bonding interactions, the overall outcome of the catalytic reaction can be improved. For this purpose, it is ideal for the reaction solvent to also act as the required hydrogen-bonding molecules. ${ }^{5}$ While there are many solvents capable of forming hydrogen bonds, with water being the one in biological systems, perfluorinated alcohols such as HFIP are attractive options for organic synthesis. ${ }^{14}$ HFIP has been known to mediate a wide range of reactions as a highly ionizing solvent with excellent hydrogen 
bonding capability, yet, its unique role in catalysis remains poorly understood. ${ }^{15}$ Simple and mildly Brønsted acidic catalysts with multiple hydrogen bond acceptor groups, such as carboxylic acids or sulfonic acids, could become suitable experimental models to further explore the concept of catalyst activation by hydrogen-bonding networks.

To study such novel catalyst systems, we embarked on the investigation of their efficiency on the carbonyl olefin metathesis (COM) reaction. ${ }^{16,17}$ The COM reaction has been identified as an attractive replacement to overcome challenges in traditional approaches for the olefination of carbonyl groups, such as pre-functionalization of substrates, reagent synthesis, or the separation of by-products from reaction mixtures. ${ }^{18,19}$ Despite recent advances in COM reactions with various Lewis acid catalysts, ${ }^{20}$ the field is still in its infancy and a generalized approach towards Brønsted acid-catalyzed COM reactions remains elusive. Up to this date, there have been only two reports on efficient Brønsted acid catalyzed COM reactions, with both of them employing elegant but very specially designed systems using fixation of the acid catalyst in a supramolecular capsule ${ }^{21}$ or within a fixed-bed in continuous flow system. ${ }^{22}$ Simple generalized methods towards COM reactions that can operate homogeneously in bulk solvent have not been reported thus far. Furthermore, the COM reaction is even more suitable for the investigation of our catalysis concept (Scheme 1B2), considering the fact that previous attempts to use superacidic catalysts such as triflic acid to catalyze COM reaction offen led to unsatisfactory or different outcomes. ${ }^{23,24}$ Therefore, the use of a hydrogen bond solvent network for catalyst activation and the demonstration of its application in the COM reaction would provide insights into the realization of new biomimetic concepts in catalysis in general and the unprecedented homogeneous Brønsted acid-catalyzed COM reactions in particular (Scheme 1B2). 
A1) Catalytic triad of serine protease bound to a boronic acid

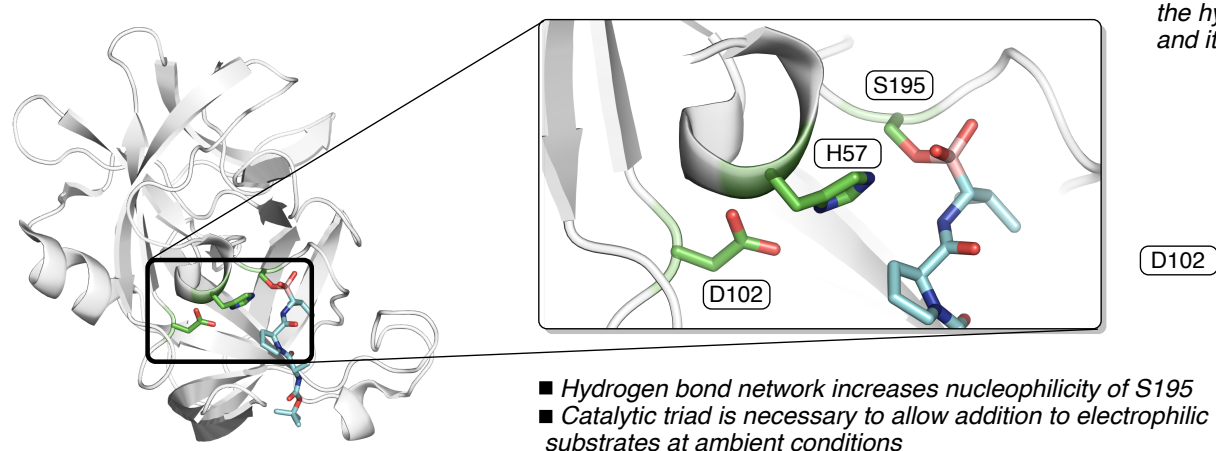

A2) Schematic representation of

the hydrogen bonding network in serine proteases and its effect on chemical reactivity

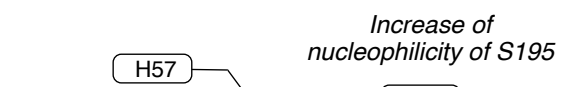

B) Hydrogen bonds in catalysis

B1) Substrate activation by hydrogen bonds

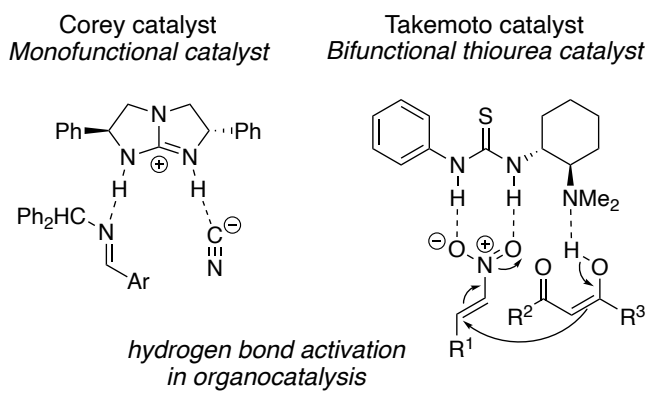

- Substrate activation via hydrogen bonds

- Preorganization of substrates via hydrogen bonds
B2) This work: Catalyst activation by hydrogen bonds

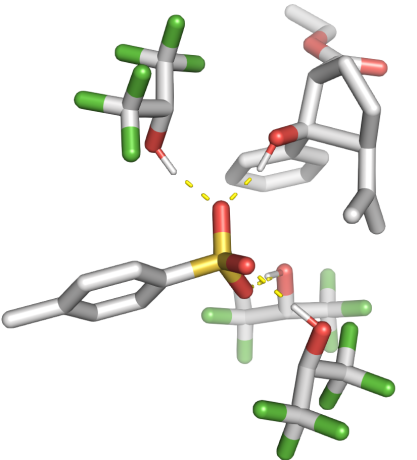

- Hydrogen bond network between catalyst and multiple molecules of HFIP - Increased catalytic efficiency of Bronsted acid catalyst

Scheme 1. Hydrogen-bonding complexation with solvent activates Brønsted acid catalysts for the promotion of otherwise challenging chemical transformation. ${ }^{11}$

\section{Reaction optimization and mechanistic investigations}

To probe our hypothesis on hydrogen bond network-assisted, Brønsted acid-catalyzed COM reactions, we studied the influence of solvent on the reaction substrate 1a using pTSA as a simple, mildly acidic and readily available Brønsted acid catalyst. Pleasingly, the reaction worked optimally with 10 mol\% of pTSA catalyst in $100 \mu \mathrm{L}$ HFIP for the $0.2 \mathrm{mmol}$ scale reaction, giving the product $2 \mathrm{a}$ in $80 \%$ yield after 4 hours at ambient temperature (entry 1, Table 1). ${ }^{25}$ Solvents such as 1,2-dichloroethane (DCE), iPrOH or linear fluorinated alcohols, which are weaker hydrogen-bonding agents than HFIP, proved to be inefficient (entries 2-6, Table 1). Our ${ }^{1} \mathrm{H}$ NMR studies on the perturbation of the pTSA acidic proton signal in the present of a varying amount of HFIP showed clear evidence of such a hydrogen-bonding network, and this effect was stronger with HFIP than iPrOH or TFE (see page S4-S7 in the experimental SI for further details). Furthermore, the use of a squaramide or a thiourea catalyst as hydrogenbonding donors did not lead to any productive outcomes either (entries 7-8), thus demonstrating the importance of HFIP and the formation of a strong hydrogen bond network to enhance catalytic efficiency of pTSA and improve the efficiency of the COM reaction. In the absence of catalyst, no reaction was observed (entry 9) and lower catalyst loading was detrimental to the reaction efficiency 
(entry 10). pTSA was superior to a range of other Brønsted acids, including strong acids such triflic acid (TfOH) as well as $\mathrm{HCl}$ or trifluoroacetic acid (entries 11-13), highlighting the special role of HFIP in mediating the COM reaction with a mildly acidic catalyst. It should be noted here again that previous attempts using triflic acid to catalyze COM reactions offen led to different outcomes. ${ }^{23,24}$ It was curious that reduced reaction efficiencies were observed for more concentrated or diluted reaction mixture (entries 14-16). Nevertheless, the optimal conditions developed here are milder and more practical than previous reports on other Brønsted acid catalyzed COM systems, which used more complicated reaction setups, elevated temperatures and longer reaction times. ${ }^{21,22}$

Table 1. Optimization of the HFIP-promoted Brønsted acid-catalyzed COM.

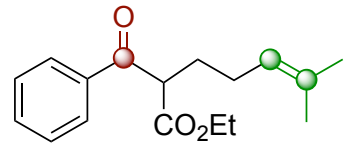

1a

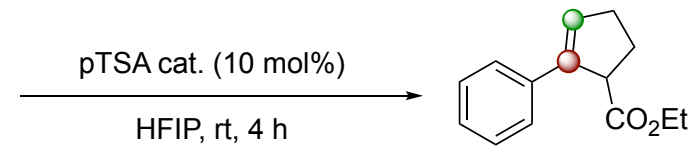

$2 a$

\begin{tabular}{|c|c|c|}
\hline Entry $^{[a]}$ & Variations from optimal conditions $^{[b]}$ & Yield $^{[c]}$ \\
\hline 1 & None (HFIP = $100 \mu \mathrm{L}$ ) & $80 \%$ \\
\hline 2 & Neat & n.p. \\
\hline 3 & DCE instead of HFIP & n.p. \\
\hline 4 & iPrOH instead of HFIP & n.p. \\
\hline 5 & TFE $\left(\mathrm{CF}_{3} \mathrm{CH}_{2} \mathrm{OH}\right)$ instead of HFIP & $15 \%$ \\
\hline 6 & $\mathrm{CF}_{3} \mathrm{CF}_{2} \mathrm{CH}_{2} \mathrm{OH}$ instead of HFIP & n.p. \\
\hline 7 & Catalyst $\mathbf{A}$ or $\mathbf{B}(10 \mathrm{~mol} \%)$ instead of pTSA, in HFIP & n.p. \\
\hline 8 & pTSA and catalyst $\mathbf{A}$ or $\mathbf{B}$ ( 10 mol\%, instead of HFIP), in DCE & n.p. \\
\hline 9 & Absence of pTSA & n.p. \\
\hline 10 & pTSA (5 mol\%) & $73 \%$ \\
\hline 11 & TfOH (10 mol\%) instead of pTSA & $66 \%$ \\
\hline 12 & $\mathrm{HCl}(10 \mathrm{~mol} \%)$ instead of pTSA & traces \\
\hline 13 & TFA (10 mol\%) instead of pTSA & traces \\
\hline 14 & HFIP $(50 \mu \mathrm{L})$ & $56 \%$ \\
\hline 15 & HFIP $(75 \mu \mathrm{L})$ & $62 \%$ \\
\hline 16 & HFIP $(200 \mu \mathrm{L})$ & $60 \%$ \\
\hline
\end{tabular}

[a] Reaction conditions: 1a $(0.2 \mathrm{mmol})$, pTSA (10 mol\%), HFIP (100 $\mu \mathrm{L})$ at $\mathrm{rt}$ for $4 \mathrm{~h}$. [b] For further details on optimization studies, see pages S8-S9 in the experimental SI. [c] Yield based on ${ }^{1} \mathrm{H}$ NMR integration using methyl benzoate as an internal standard, n.p. $=$ no product. 
For further understanding of the reaction mechanism and the role of HFIP and the hydrogen bond network on the reaction, we carried out a series of kinetic studies with substrate $1 \mathrm{a}$ and 5 mol\% of pTSA in varying amount of HFIP from 1 to 4 equivalents with respect to 1a (Figure 1). The conversion of 1a was monitored by ${ }^{1} \mathrm{H}$ NMR spectroscopy over time (see pages S10-S11 in the experimental SI for more details). The kinetic data was analyzed and showed that the reaction order in HFIP was 3.2 (Figure 1), which suggested that only a small number of HFIP solvent molecules were involved in the rate determining step of the COM reaction under investigation.
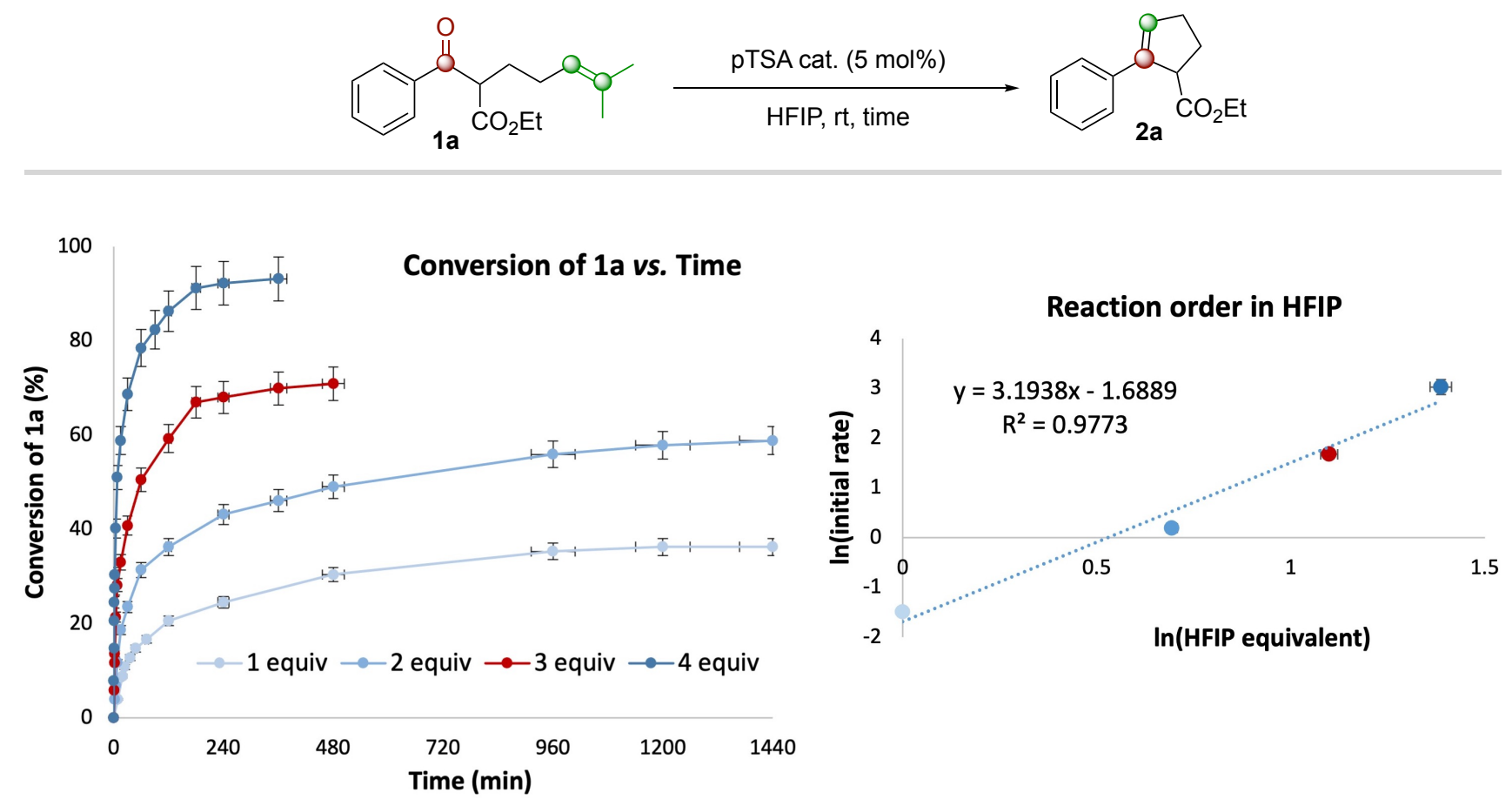

Figure 1. Kinetic studies of the conversion of $1 \mathbf{a}$ to product 2a with different amounts of HFIP (See pages S10-S11 in the

To understand experimental reaction kinetics and to rationalize the influence of HFIP on the reaction mechanism, we next embarked on computational studies on the pTSA-catalyzed reaction of 1a (Scheme 2). First, we examined an implicit solvent model for $\mathrm{HFIP}^{26}$ that does not allow for interaction of solvent molecules with substrate and/or catalyst (Scheme 2a, grey energy profile). Second, we used a combination of explicit solvent molecules and an additional implicit solvent model. For this, we added - in accordance to the previous experimental observation - three explicit molecules of HFIP to the calculation to account for the formation and influence of a hydrogen bond network between solvent molecules and catalyst (Scheme 2a, dark blue energy profile). Disregarding of the solvent model used, the calculations show that this COM reaction proceeds via the same elementary reactions steps and initiates via an intramolecular $\mathrm{C}-\mathrm{C}$ bond formation reaction, followed by oxetane formation, ring opening and elimination reaction to provide olefin product $2 \mathrm{a}$. Each of these four elementary reaction 
steps is catalyzed by pTSA, i.e. (i) activation of the carbonyl group in the C-C bond formation step, (ii) and (iii) hydrogen bond interactions during ring-closing and ring-opening of the oxetane and (iv) activation of the carbonyl group that leads to cleavage of the acetone by-product and release of the COM product, respectively.

While the reaction pathway is not altered by the introduction of the hydrogen bond network and with/without the hydrogen bond network the oxetane ring formation remains the rate-determining step. The hydrogen bond network has however a significant influence on the activation free energy along the path of the COM reaction (Scheme 2a, grey vs. blue profile). For instance, the barrier of the initial C-C bond formation is reduced from 23.9 to $12.9 \mathrm{kcal} / \mathrm{mol}$ in the presence of 3 molecules of HFIP (Scheme 2a, TS1). Similarly, the introduction of 3 molecules of HFIP leads to a significant reduction of the activation free energy of the oxetane formation, which was identified as rate-determining step with an activation free energy of $30.2 \mathrm{kcal} / \mathrm{mol}$ without HFIP and $14.8 \mathrm{kcal} / \mathrm{mol}$ in the presence of 3 molecules of HFIP, respectively. In the second stage of the reaction, the oxetane intermediate INT2 is ring-opened in the presence of the pTSA catalyst. The introduction of additional molecules of HIFP similarly leads to a marked reduction of the activation free energies, e.g. from 25.3 to $13.9 \mathrm{kcal} / \mathrm{mol}$ for the formation of the carbocation intermediate INT3 upon introduction of three explicit molecules of HFIP. Thus, the formation of a hydrogen bond network of 1a, pTSA and three molecules of HFIP leads to a significant lowering of the activation free energy and renders the room temperature COM reactions with simple Brønsted acids possible.

Next, we performed a closer examination of the influence of the hydrogen bond network with different alcohol solvents on the activation of the pTSA catalyst (Scheme $2 \mathrm{~b}$ ). First, we examined $i$ PrOH as a close analogue of HFIP to model the influence of a weak hydrogen bond donor (Scheme $2 b$, grey). In this case relatively high activation free energies were observed, which are comparable to calculations with an implicit solvent (cf. Scheme 2a). The activation free energy of the rate-determining step was calculated with $28.4 \mathrm{kcal} / \mathrm{mol}$, which is too high to proceed at room temperature with reasonable efficiency. Next, we examined trifluoroethanol as a model for an increased ability to form hydrogen bond networks (Scheme 2b, light blue). In comparison to $\mathrm{PrOH}$, the hydrogen bond network of solvent and catalyst results in a significant reduction of the activation free energy of all transition states. However, only in the case of the strong hydrogen bond donor HFIP (Scheme 2a and $2 b$, dark blue), the activation free energy for all reaction steps are significantly reduced to enable for efficient COM reaction. Further calculations concerned the analysis of the influence of the stoichiometry of HFIP and catalyst. This analysis reveals that three molecules of HFIP form an optimal hydrogen bond network 
and allow for the COM reaction to proceed under mild conditions (Scheme 2c), which can be attributed to the presence of three oxygen atoms in PTSA that are required for hydrogen bonding to three molecules of HFIP (Scheme 2d,e). These calculations now show that HFIP engages in the formation of hydrogen bonding interactions with the pTSA catalyst that results in an encapsulation of the catalyst within a hydrogen bond network. This hydrogen bond network thus alters properties of the pTSA catalyst and consequently the transition state energies for each step.

a) Influence of hydrogen bond network on the reaction profile of the COM reaction

Evaluation of implicit solvent model for HFIP (grey) and a combined explicit-implicit solvent model (dark blue). Structures presented are representative for the combined explicit-implicit solvent model
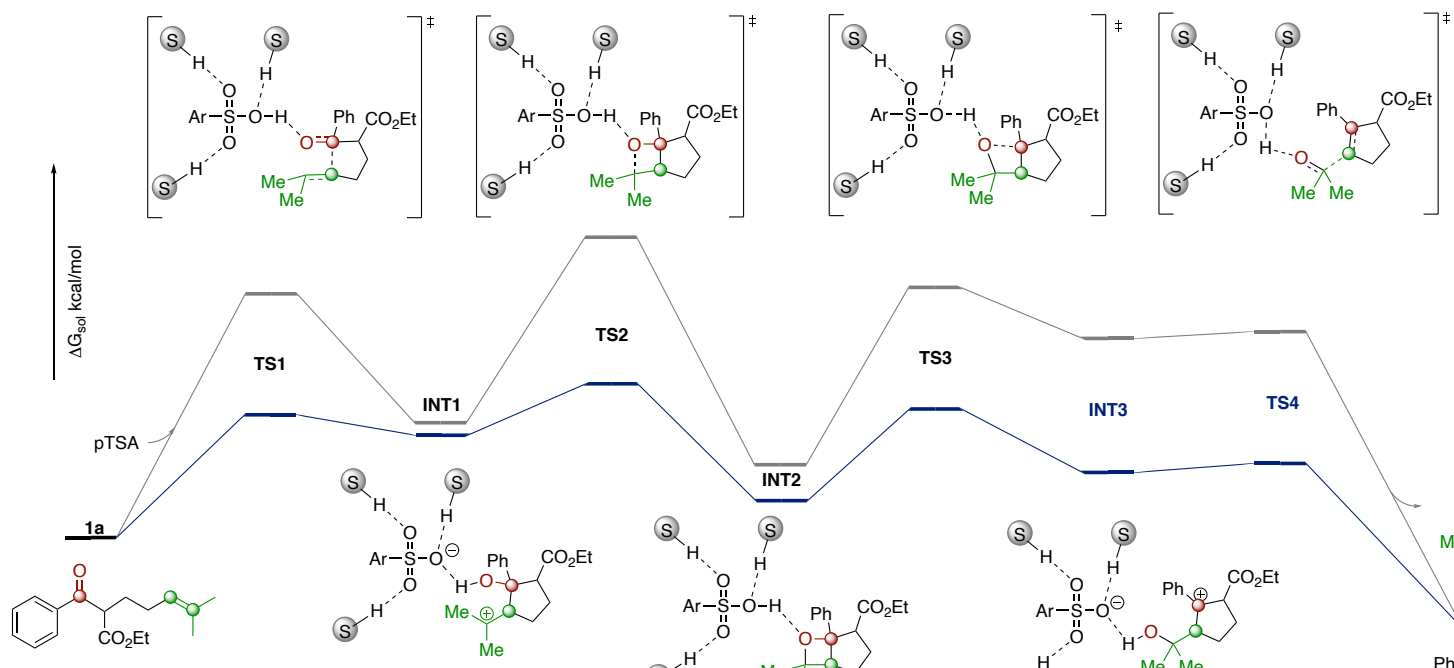
Evaluation of the influence of three different alcohols.

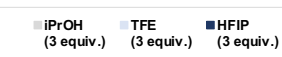

C-c bond formation Oxetane formation

Ring opening TS3

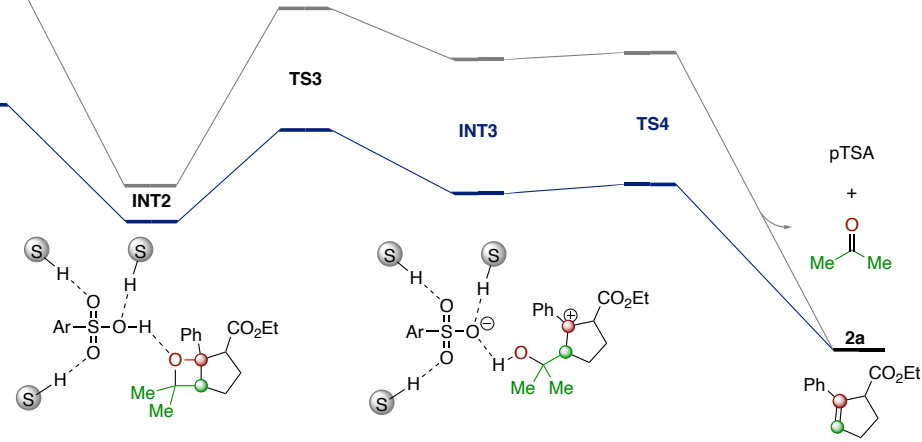

c) Influence of the stoichiometry Influence of number of explicit HFIP molecules

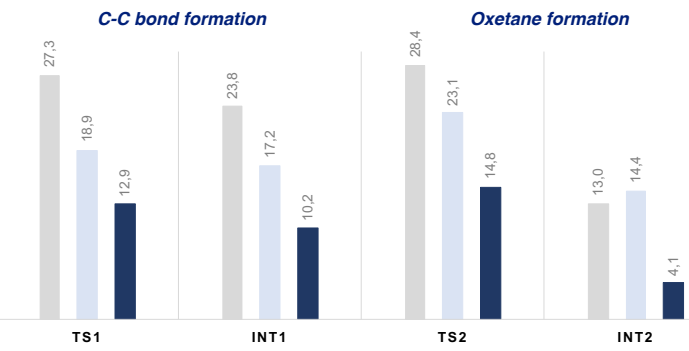

d) Comparison of a selected transition state Transition state for oxetane formation (TS2)

e) Comparison of a selected intermediate

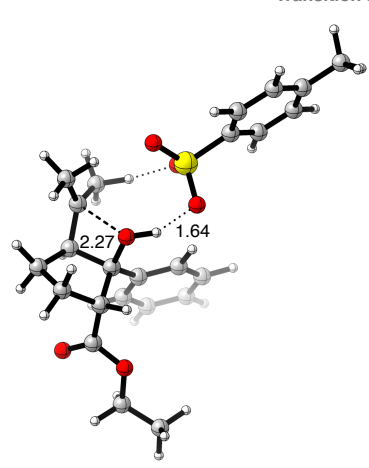

cyclic transition state

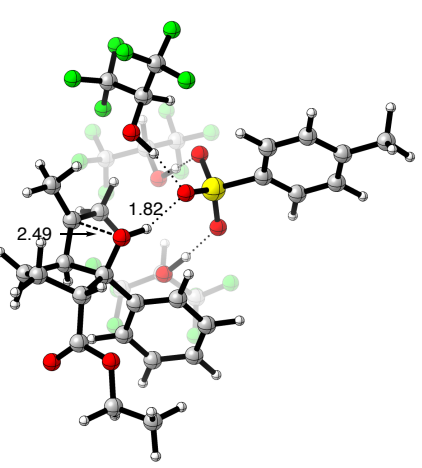

acyclic transition state

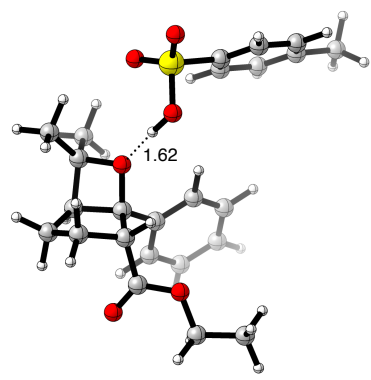

interaction via hydrogen bonding

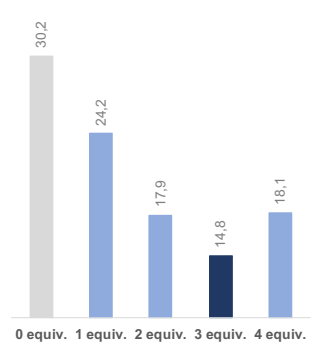

0 equiv. 1 equiv. 2 equiv, 3 equiv, 4 equiv.

Scheme 2. Theoretical calculations on the PTSA-catalyzed COM reaction and the influence of HFIP hydrogen bond networks. Level of theory: B3LYP-D3BJ/def2-tzvp (SMD = HFIP)//B3LYP/def2-svp. 
171

172

173

174

175

176

177

178

179

180

181

182

183

184

185

The optimized conditions developed in Table 1 were then applied to a range of intramolecular COM substrates (Scheme 3b). $\alpha$-Substituted ketoester substrates reacted smoothly to form their corresponding cyclopentene products in moderate to high yields (2a-h). For some substrates, the isomerized cyclopentenes were obtained as major products (2'e and $\mathbf{2} \mathbf{h}$ ), which was expected in this Brønsted acidic environment. The reaction worked particularly well to form indene derivatives (2i-k and $2^{\prime}$ I), which can be attributed to the stability of the conjugate indene ring that formed (Scheme 3). Five-membered $\mathrm{N}$-heterocyclic products could also be formed by this method in good to high yields, although the reactions on non $\alpha$-substituted systems $(2 m-n)$ were less efficient than those of $\alpha$ substituted ones (20-p). The formation of six-membered $\mathrm{N}$-heterocyclic products was more challenging, which required higher catalyst loading in more diluted reaction but still only led to moderate reaction outcomes ( $2 q$ and $\mathbf{2 r}$, Scheme $3 b)$.

As discussed earlier, the directed Brønsted acid catalyzed COM reaction in homogeneous conditions is often problematic in that several side processes such as carbonyl-ene, Prins or interrupted carbonylolefin metathesis reactions. ${ }^{23,24}$ As our pTSA/HFIP catalytic system marked the first time COM reactions can be carried out in this manner without much of those issues, we would like to expand the work to investigate the scope of its catalytic activity on analogous cyclization reactions. We decided to select a series of aromatic ketones with an unsaturated side chain $(\mathbf{1}, \mathbf{3}, \mathbf{5}, \mathbf{7}, \mathbf{9}$ Scheme 3$)$ and subjected them to the pTSA/HFIP catalytic conditions. The $\varepsilon, \zeta$-unsaturated ketone substrates in Scheme $3 a$ were modeled after Schindler's interrupted COM reaction substrates. ${ }^{23}$ They have unsaturated side chains with one more carbon than the $\operatorname{COM} \delta, \varepsilon$-unsaturated ketone substrates in Scheme $3 b$. The $\gamma, \delta$ unsaturated ketone substrates in Scheme $3 \mathrm{c}$ can be considered one $\mathrm{CH}_{2}$ truncated versions of the COM substrates. The alkenyl and alkynyl keto subtrates in Scheme $3 \mathrm{~d}$ and $3 \mathrm{e}$ bear slightly different unsaturated side chains but can be considered synthetic equivalents of the ones in Scheme 3c.

Most of these tested substrates cyclized under our pTSA/HFIP catalytic conditions to give the corresponding products $(\mathbf{2}, \mathbf{4}, \mathbf{6}, \mathbf{8}$, Scheme 3$)$ in moderate to high yields within four hours at ambient temperature. Some cyclization processes required to be carried out at $50{ }^{\circ} \mathrm{C}$ to afford satisfactory outcomes, as indicated by product yields in parentheses. It is interesting to see that electron-donating substituent such as $\mathrm{OMe}$ or electron-withdrawing substituent such as $\mathrm{NO}_{2}$ can have completely opposite effects on the outcomes of these 6-endo-trig (Scheme 3c), 5-exo-dig (Scheme 3d) and 5-exotrig (Scheme 3e) cyclization reactions. 


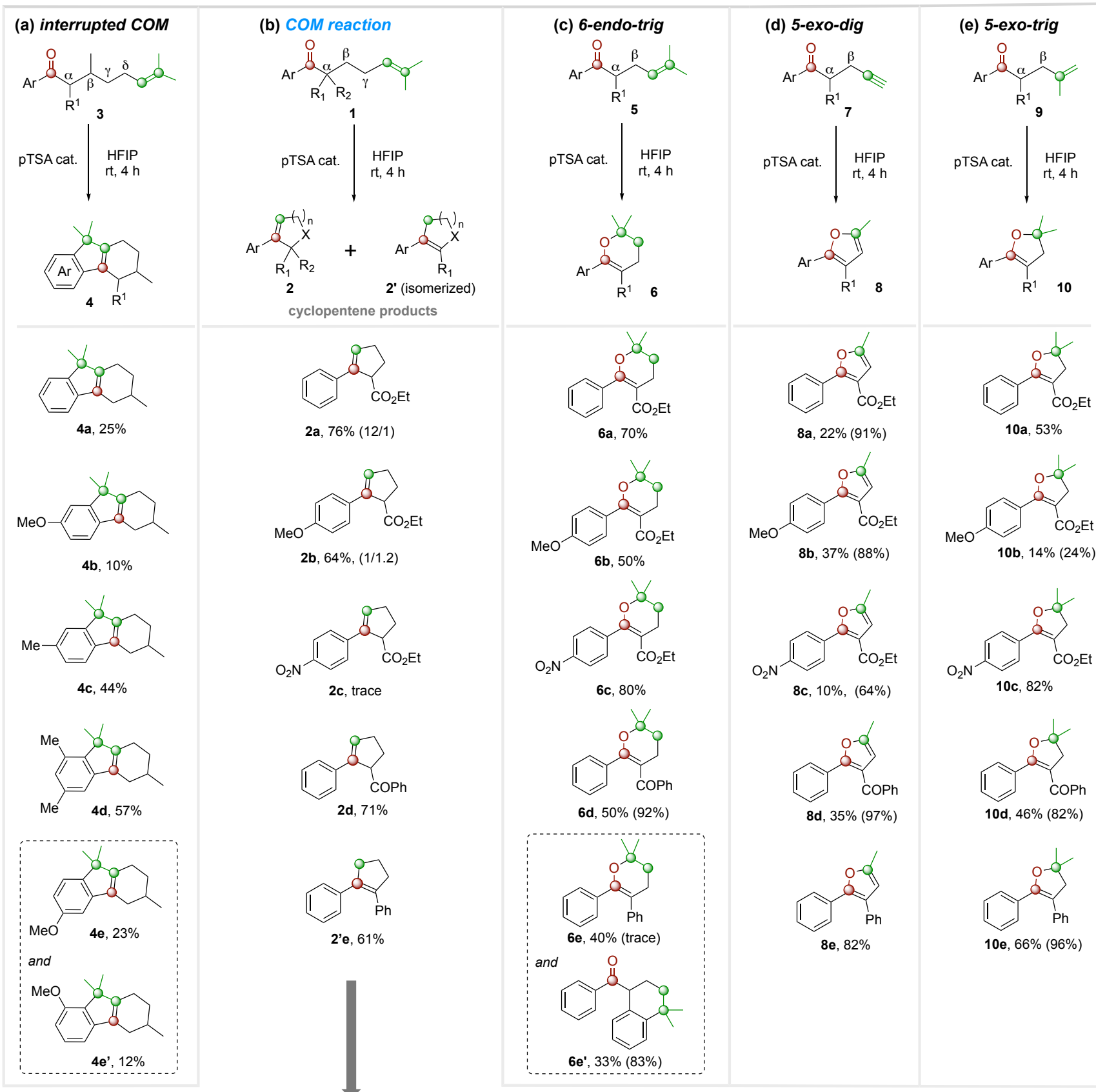<smiles>[R]c1ccccc1C1CCCO1</smiles>

2f, $33 \%(20 / 1)$

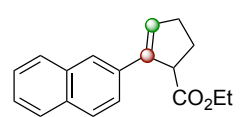

2g, $80 \%(11 / 1)$

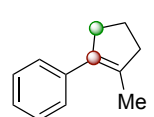

2'h, 30\%

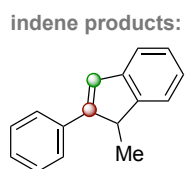

2i, $70 \%(1 / 1.25)$

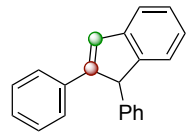

2j, $95 \%$

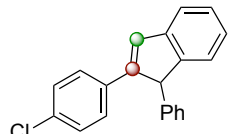

2k, $91 \%$

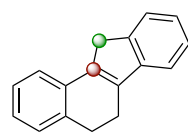

2'।, $92 \%$

five-membered $N$-heterocylic products:

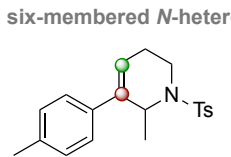

$2 q, 28 \%$

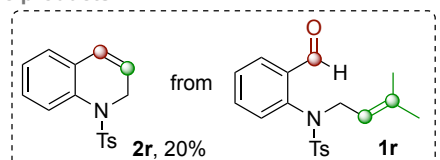

Ts $2 r, 20 \%$

Scheme 3. Substrate scope of COM reaction and analogous cyclization reactions under PTSA/HFIP catalytic conditions: (unless otherwise specified) substrate $(0.2 \mathrm{mmol})$, pTSA (10 mol\%), HFIP (100 $\mu \mathrm{L})$ at $\mathrm{rt}$ for $4 \mathrm{~h}$. For the formation of product 4, reactions were carried out in PhCl/HFIP $(1.8 \mathrm{~mL} / 0.2 \mathrm{~mL})$ for $18 \mathrm{~h}$. Yields are of isolated products. Yields in parentheses are of reactions carried out at $50^{\circ} \mathrm{C}$. Ratio in parentheses are of products 2 to $\mathbf{2}^{\prime}$. 
When there was an aromatic substituent at the alpha position, the 6-endo-trig cyclization was not the only predominant reaction pathway (Scheme 3c, product $\left.6 \mathrm{e} / 6 \mathrm{e}^{\prime}\right)$. The substrate could also cyclize in a Friedel-Crafts alkylation fashion to form tetrahydronaphthalene product $6 \mathbf{e}^{\prime}$, which became the single major product at elevated temperature. This reaction pathway is directly relevant to the formation of products $\mathbf{4}$ in Scheme 3a, where presumably the carbocation intermediate from a COM process also underwent Friedel-Crafts alkylation reaction onto the adjacent aromatic ring to form the tricyclic system. ${ }^{23}$ Such interrupted COM reaction is possible for this type of substrate but not the typical COM substrate (Scheme $3 \mathrm{~b}$ ), which can be attributed to the conformational arrangement of the initially formed six-membered ring. The efficiency of the interrupted COM reaction mediated by our pTSA/HFIP, albeit not fully optimized, was slightly lower than that of the earlier study with TfOH catalyst by Schindler and co-workers. ${ }^{23}$ We also observed some direct addition of HFIP to the olefin moieties for some substrates with the $\alpha$-substituent being ester, ketone or phenyl groups (see pages S48-S49 in the experimental SI for details). It posed the question of how different does HFIP make those pTSA-catalyzed reactions in Scheme 3 in comparison to a normal organic solvent. Furthermore, would the super Brønsted acidic TfOH overcome the need for the 'magical effect' of HFIP to efficiently promote those cyclization reactions in a normal organic solvent?

Thus, we decided to carry out a comparative study where we performed two of each type of the 6endo-trig cyclization, the COM reactions and the interrupted COM reactions in different sets of conditions with pTSA/HFIP and TfOH/DCE (Table 2, for further details on these studies and also the reaction performances on the 5-exo-trig, 5-exo-dig cyclizations, see page S59 in the experimental SI). Interestingly, we observed clear differences in reaction efficiency. pTSA/HFIP system proved to be a lot more superior than TfOH/DCE in the COM cyclization (products $\mathbf{2 a}$ and 2'e). For the 6-endo-trig cyclization (products $6 a$ and $6 \mathrm{e} / 6 \mathrm{e}^{\prime}$ ), TfOH/DCE was slightly inferior to pTSA/HFIP, especially when it came to the formation of Friedel-Crafts type product $6 e^{\prime}$ at elevated temperature. Similar catalyst/solvent-reactivity relationship was observed for the interrupted COM products (4a and $4 \mathbf{e}$ ). These results once again confirmed the very important role of HFIP solvent and formation of hydrogen bond networks in these Brønsted acid catalyzed reactions. 
Table 2. Comparison of reactions using two different catalytic systems: pTSA/HFIP and TfOH/DCE ${ }^{[a]}$

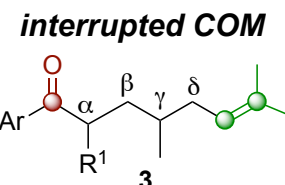

cat. $\downarrow^{3}$ solvent
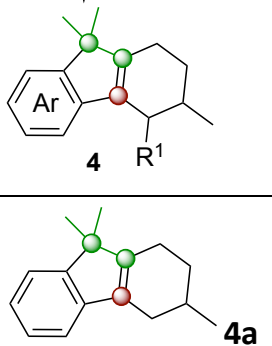

Product

PTSA/HFIP

TfOH/DCE

Product

$6 e$

$\begin{array}{lcc}\text { pTSA/HFIP } & 58 \% & 77 \%^{[\mathrm{b}]} \\ \text { TfOH/DCE } & 37 \% & 24 \% \%^{[\mathrm{b}]}\left(64 \%^{[\mathrm{b}]}\right)\end{array}$

6-endo-trig

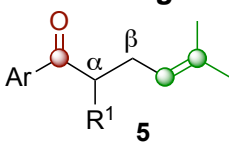

cat. solvent

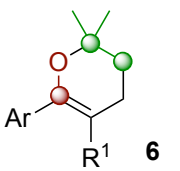

2 2' (isomerized)

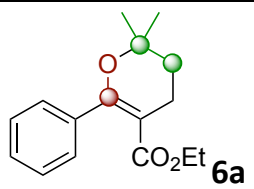

$73 \%$

$67 \%$

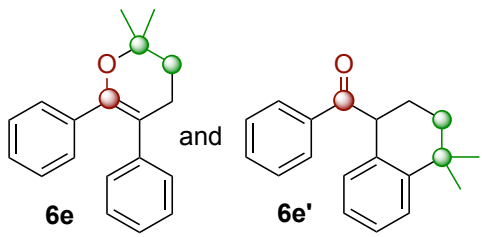

5e: $42 \%$ (trace); $5 \mathbf{e}^{\prime}: 35 \%$ (83\%)

5e: $45 \%$ (7\%); $5 \mathbf{e}^{\prime}$ : traces (28\%)

[a] Reaction condition: Substrate $(0.2 \mathrm{mmol})$, pTSA or TfOH (10 mol\%), HFIP or DCE (100 $\mu \mathrm{L})$ at RT for $4 \mathrm{~h}$. Yields in parentheses are of reactions carried out at $50{ }^{\circ} \mathrm{C}$. Yields were determined by ${ }^{1} \mathrm{H}$ NMR integration using mesitylene as an internal standard. [b] Overall yields of two olefin isomers $\mathbf{2} / \mathbf{2}^{\prime}$.

The above comparative study on the influence of the carbon skeleton on the reaction outcome encouraged us to further examine and rationalize this intriguing divergent reactivity. It is of particular interest to understand current limitations ${ }^{20}$ in carbonyl olefin metathesis ring-closing reactions and the specific reactivity for the preferential formation of cyclopentenes. The corresponding cyclobutenes or cyclohexenes are not favored products from COM cyclization and highly specialized catalysts are required in scarce number of reports for the latter. ${ }^{27,28}$ We therefore carried out computational studies on the COM reaction pathway and all other reaction pathways observed for different chain length of the alkenyl carbon skeleton (Scheme 4). The analysis of the first reaction step showed a distinct effect of the carbon chain length on the activation free energy for C-C bond formation (TS1). This step is energetically favored for the hexene $(1, n=2)$ and heptene $(3, n=3)$ substrates, while being energetically highly unfavorable for the shorter pentene derivative $(5, n=1)$ due to the high ring strain of the putative 1-oxo-bicyclo-[2.2.0]-hexane intermediate (TS1-1) (Scheme 4, dark blue). Instead, 5 preferentially undergoes a 6-endo dig cyclization reaction via TS5 to give pyrane 6a (Scheme 4, green). The analysis of similar cyclization pathways for hexene $(\mathbf{1}, n=2)$ and heptene $(3, n=3)$ substrates 
showed that such cyclization is indeed possible, yet unfavored due to the formation of larger ring systems and transannular interactions within such ring systems. ${ }^{29}$

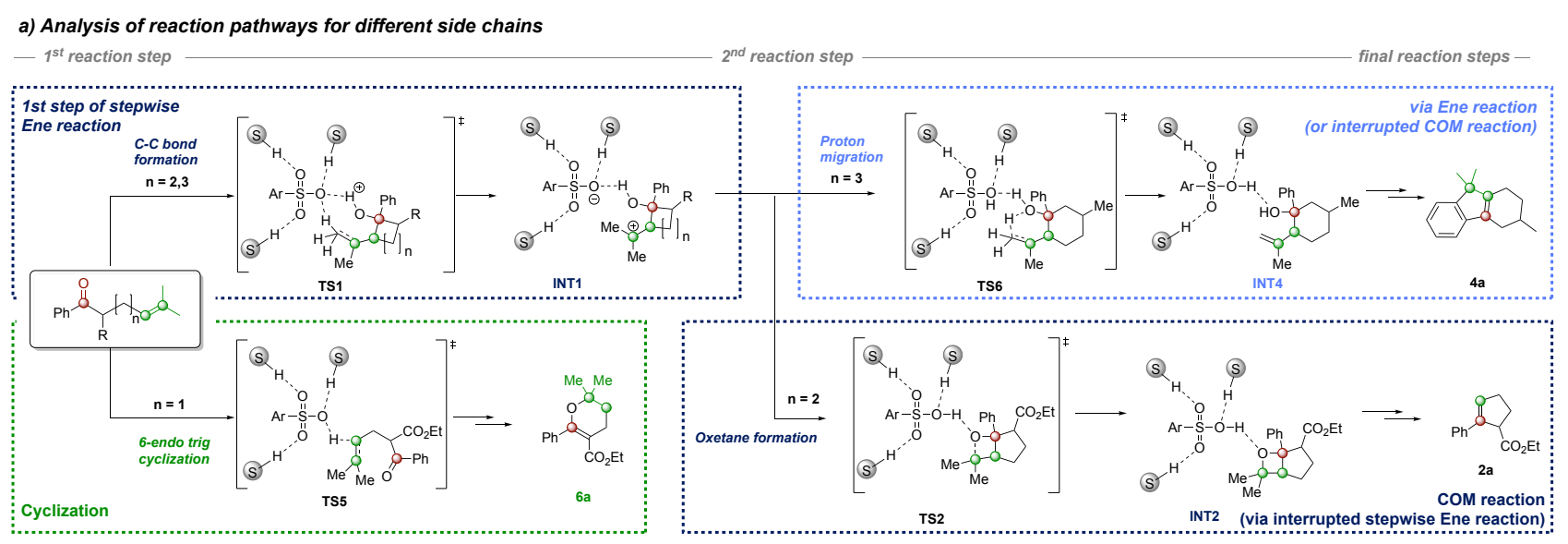

b) Comparison of activation free energy and transition state structures for different chain length for key reaction steps - $1^{\text {st }}$ reaction step
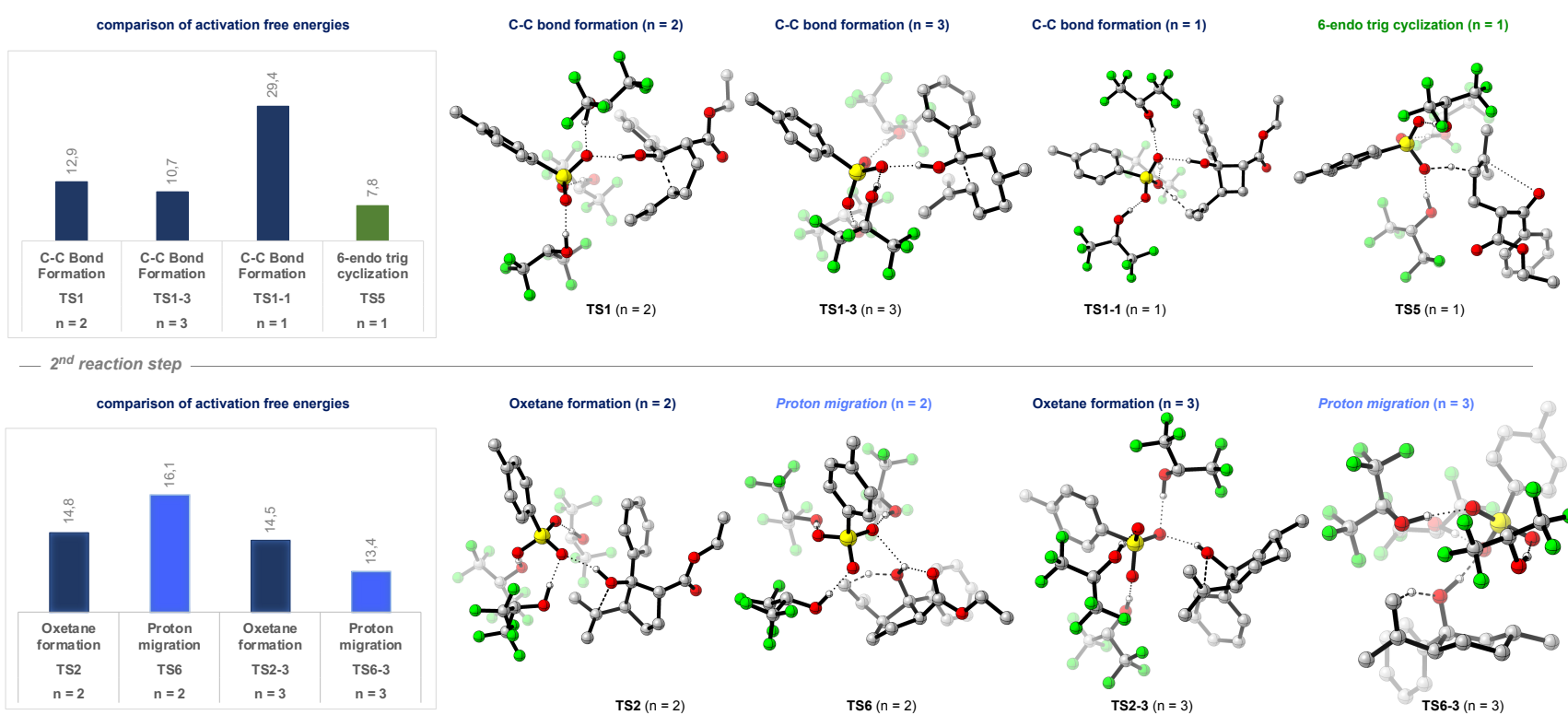

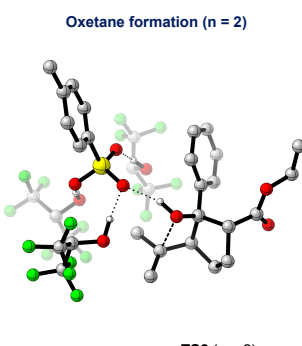

TS2 ( $(n=2)$

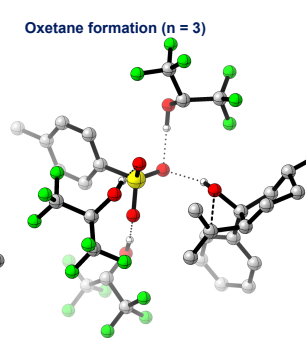

TS2-3 $(n=3)$

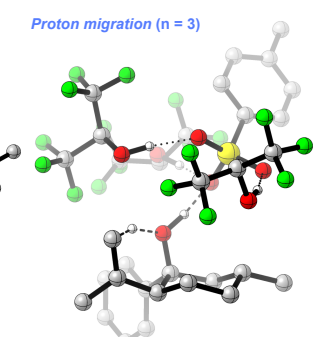

TS6 $(n=2)$

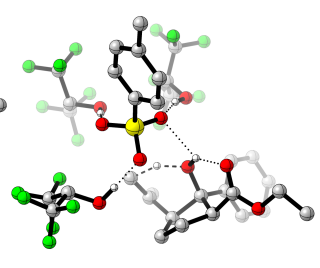

Scheme 4. Comparison of the influence of the alkenyl chain length on the reaction outcomes.

The second reaction step then rationalizes for the divergent reactivity of the hexene $(\mathbf{1}, \mathrm{n}=\mathbf{2})$ and heptene $(3, n=3)$ substrates. Both substrates can potentially undergo a proton migration reaction ${ }^{30}$ via the bicyclic transition state TS6, which results in the product of a classic Ene reaction (INT4) via a stepwise reaction mechanism. Following the stepwise Ene reaction, the tricyclic reaction product $6 a$ (Scheme 4, light blue) is formed, which is often referred to as the product of an interrupted COM reaction. The interruption of the Ene reaction pathway however, allows the formation of the bicyclic oxetane intermediate (INT2) via transition state TS2 that ultimately leads to COM reaction (Scheme 4, dark blue). Thus, the initial steps of a COM reaction can also be regarded as an interrupted stepwise 
271 bicyclic oxetane intermediate INT2 is conformationally accessible due to the envelope conformation 272 of 5-membered rings. In the case of heptenes (3), this pathway cannot be accessed as the six273 membered ring needs to adapt an unfavorable twist boat conformation. Small differences in the 274 energy of transition states that result from conformational restriction of bicyclic transition states 275 and/or intermediates thus open a divergent reactivity that can lead to cyclization, carbonyl olefin 276 metathesis or Ene reaction.

\section{Conclusion}

278 In summary, we report on a combined experimental and computational study on the activation of 279 catalysts by hydrogen bonding interaction. We show that HFIP can act as a hydrogen bond donor to 280 enhance the catalytic efficiency of simple Brønsted acid catalysts by stabilization of all transition states 281 and intermediates along the reaction pathway. This mode of activation could successfully be employed 282 to allow for a novel and practical method for the direct Brønsted acid catalyzed carbonyl-olefin 283 metathesis reaction. Interesting insights into the effect of the alkenyl moiety chain length on the reaction outcomes were also revealed, which give the rationalization for the current ring-size limitation of COM cyclization reaction products. These results will not only advance the catalytic scope of the COM reaction further into homogeneous Brønsted acid catalysis but also pave the way for further investigations and applications of hydrogen bonding network assisted catalysis in organic synthesis. 


\section{ASSOCIATED CONTENT}

\section{Supporting Information}

The Supporting Information is available free of charge: Experimental details and spectroscopic data for all products, full Gaussian reference, Cartesian coordinates, electronic and free energies.

\section{AUTHOR INFORMATION}

\section{Materials and Correspondence}

*RMK. E-mail: rene.koenigs@rwth-aachen.de

*TVN. E-mail: t.v.nguyen@unsw.edu.au

\section{Author Contributions}

The manuscript was written through contributions of all authors. TAT carried out all experimental work; TVN and RMK conceived the ideas and designed the project. CP and RMK carried out all computational studies. All authors have given approval to the final version of the manuscript.

\section{CONFLICTS OF INTEREST}

There is no conflicts of interest to declare.

\section{ACKNOWLEDGMENTS}

This work was funded by the Australian Research Council (grant FT180100260 to TVN and DP200100063 to TVN and RMK).

\section{REFERENCES}

1 Anslyn, E. V. \& Dougherty, D. A. in Modern Physical Organic Chemistry, pp 145-204 (University Science Books, 2006).

2 Jeffrey, G. A. \& Saenger, W. in Hydrogen Bonding in Biological Structures, pp 167-422 (Springer, 1991).

3 Meeuwissen, J. \& Reek, J. N. H. Supramolecular catalysis beyond enzyme mimics. Nature Chemistry 2, 615-621, doi:https://doi.org/10.1038/nchem.744 (2010).

4 Herschlag, D. \& Pinney, M. M. Hydrogen Bonds: Simple after All? Biochemistry 57, 3338-3352, doi:https://doi.org/10.1021/acs.biochem.8b00217 (2018). 

Computational Molecular Science 10, e1477, doi:https://doi.org/10.1002/wcms.1477 (2020). 6 Dai, S. et al. Low-barrier hydrogen bonds in enzyme cooperativity. Nature 573, 609-613, doi:https://doi.org/10.1038/s41586-019-1581-9 (2019).

3207

7 Eder, U., Sauer, G. \& Wiechert, R. New Type of Asymmetric Cyclization to Optically Active

Steroid CD Partial Structures. Angewandte Chemie International Edition in English 10, 496-497,

3238
doi:https://doi.org/10.1002/anie.197104961 (1971).

Hajos, Z. G. \& Parrish, D. R. Asymmetric synthesis of bicyclic intermediates of natural product chemistry. The Journal of Organic Chemistry 39, 1615-1621, doi:https://doi.org/10.1021/jo00925a003 (1974).

9 Schreiner, P. R. Metal-free organocatalysis through explicit hydrogen bonding interactions. Chemical Society Reviews 32, 289-296, doi:https://doi.org/10.1039/B107298F (2003).

10 Knowles, R. R. \& Jacobsen, E. N. Attractive noncovalent interactions in asymmetric catalysis: Links between enzymes and small molecule catalysts. Proceedings of the National Academy of Sciences 107, 20678-20685, doi:https://doi.org/10.1073/pnas.1006402107 (2010).

11 Image of 1P01 (Bone, R., Agard, D.A. Serine protease mechanism. structure of an inhibitory complex of ALPHA-LYTIC Protease and a tightly bound peptide boronic acid, doi: http://doi.org/10.2210/pdb1P01/pdb (1990); and literature: Bone, R., Shenvi, A.B., Kettner, C.A., Agard, D.A. Biochemistry 26, 7609-7614, doi: http://dx.doi.org/10.1021/bi00398a012 (1987)) created with PyMOL (The PyMOL Molecular Graphics System, Version 2.5.0 for Macintosh, Schrödinger, LLC). 12 Hedstrom, L. Serine Protease Mechanism and Specificity. Chemical Reviews 102, 4501-4524, doi:https://doi.org/10.1021/cr000033x (2002).

13 Schreiner, P. R. Cooperativity Tames Reactive Catalysts. Science 327, 965-966, doi:https://doi.org/10.1126/science.1186764 (2010).

14 Colomer, I., Chamberlain, A. E. R., Haughey, M. B. \& Donohoe, T. J. Hexafluoroisopropanol as a highly versatile solvent. Nature Reviews Chemistry 1, 0088, doi:https://doi.org/10.1038/s41570-017$\underline{0088}(2017)$.

15 Pozhydaiev, V., Power, M., Gandon, V., Moran, J. \& Lebœuf, D. Exploiting hexafluoroisopropanol (HFIP) in Lewis and Brønsted acid-catalyzed reactions. Chemical Communications 56, 11548-11564, doi:https://doi.org/10.1039/D0CC05194B (2020).

16 Griffith, A. K., Vanos, C. M. \& Lambert, T. H. Organocatalytic Carbonyl-Olefin Metathesis. Journal of the American Chemical Society 134, 18581-18584, doi:https://doi.org/10.1021/ja309650u (2012). 
17 Ludwig, J. R., Zimmerman, P. M., Gianino, J. B. \& Schindler, C. S. Iron(III)-catalysed carbonylolefin metathesis. Nature 533, 374-379, doi:https://doi.org/10.1038/nature17432 (2016). 18 Tran, U. P. N., Oss, G., Pace, D. P., Ho, J. \& Nguyen, T. V. Tropylium-promoted carbonyl-olefin metathesis reactions. Chemical Science

9, 5145-5151, doi:https://doi.org/10.1039/C8SC00907D 353 (2018).

35419 Tran, U. P. N. et al. Carbonyl-Olefin Metathesis Catalyzed by Molecular lodine. ACS Catalysis 9, 355 912-919, doi:https://doi.org/10.1021/acscatal.8b03769 (2019).

35620 Albright, $\mathrm{H}$. et al. Carbonyl-Olefin Metathesis. Chemical Reviews, 357 doi:https://doi.org/10.1021/acs.chemrev.0c01096 (2021).

35821 Catti, L. \& Tiefenbacher, K. Brønsted Acid-Catalyzed Carbonyl-Olefin Metathesis inside a Self359 Assembled Supramolecular Host. Angewandte Chemie International Edition 57, 14589-14592, 360 doi:https://doi.org/10.1002/anie.201712141 (2018). 22 Rivero-Crespo, M. Á., Tejeda-Serrano, M., Pérez-Sánchez, H., Cerón-Carrasco, J. P. \& LeyvaPérez, A. Intermolecular Carbonyl-olefin Metathesis with Vinyl Ethers Catalyzed by Homogeneous and Solid Acids in Flow. Angewandte Chemie International Edition 59, 3846-3849, doi:https://doi.org/10.1002/anie.201909597 (2020).

23 Ludwig, J. R. et al. Interrupted carbonyl-olefin metathesis via oxygen atom transfer. Science 361, 1363-1369, doi:https://doi.org/10.1126/science.aar8238 (2018).

24 Malakar, T. \& Zimmerman, P. M. Brønsted-Acid-Catalyzed Intramolecular Carbonyl-Olefin Reactions: Interrupted Metathesis vs Carbonyl-Ene Reaction. The Journal of Organic Chemistry 86, 3008-3016, doi:https://doi.org/10.1021/acs.joc.0c03021 (2021). 25 See the experimental Supporting Information for more details.

Li, G.-X. et al. A unified photoredox-catalysis strategy for C(sp3)-H hydroxylation and amidation using hypervalent iodine. Chemical Science 8, 7180-7185, doi:10.1039/C7SC02773G (2017). 27 Djurovic, A. et al. Synthesis of Medium-Sized Carbocycles by Gallium-Catalyzed Tandem

Organic Letters

21, 8132-8137, doi:https://doi.org/10.1021/acs.orglett.9b03240 (2019).

28 Davis, A. J., Watson, R. B., Nasrallah, D. J., Gomez-Lopez, J. L. \& Schindler, C. S. Superelectrophilic aluminium(iii)-ion pairs promote a distinct reaction path for carbonyl-olefin ringclosing metathesis. Nature Catalysis 3, 787-796, doi:https://doi.org/10.1038/s41929-020-00499-5 (2020).

29 See the computational Supporting Information for more details. 
38130 Jana, S. et al. Photoinduced Proton-Transfer Reactions for Mild O-H Functionalization of 382 Unreactive Alcohols. Angewandte Chemie International Edition 59, 5562-5566, 383 doi:https://doi.org/10.1002/anie.201915161 (2020). 